\title{
On Local Aspects of Topological Transitivity and Weak Mixing in Set-Valued Discrete Systems
}

\author{
Lei Liu \\ Department of Mathematics, Shangqiu Normal University, Shangqiu, Henan 476000, China \\ Correspondence should be addressed to Lei Liu; mathliulei@yahoo.com.cn
}

Received 3 March 2013; Accepted 15 May 2013

Academic Editor: Carlo Piccardi

Copyright (C) 2013 Lei Liu. This is an open access article distributed under the Creative Commons Attribution License, which permits unrestricted use, distribution, and reproduction in any medium, provided the original work is properly cited.

\begin{abstract}
Blanchard and Huang introduced the notion of weakly mixing subset, and Oprocha and Zhang gave the concept of transitive subset and studied its basic properties. In this paper our main goal is to discuss the weakly mixing subsets and transitive subsets in setvalued discrete systems. We prove that a set-valued discrete system has a transitive subset if and only if original system has a weakly mixing subset. Moreover, we give an example showing that original system has a transitive subset, which does not imply set-valued discrete system has a transitive subset.
\end{abstract}

\section{Introduction}

Throughout this paper a topological dynamical system (abbreviated to TDS) is a pair $(X, f)$, where $X$ is a compact metric space with metric $d$ and $f: X \rightarrow X$ is a continuous map. When $X$ is finite, it is a discrete space and there is no any nontrivial convergence. Hence, we assume that $X$ contains infinitely many points. Let $\mathbb{N}$ denote the set of all positive integers and let $\mathbb{Z}_{+}=\mathbb{N} \cup\{0\}$.

Topological transitivity, weak mixing, and sensitive dependence on initial conditions (see [1-4]) are global characteristics of topological dynamical systems. Let $(X, f)$ be a TDS. $(X, f)$ is (topologically) transitive if for any nonempty open subsets $U$ and $V$ of $X$ there exists an $n \in \mathbb{N}$ such that $f^{n}(U) \cap V \neq \emptyset .(X, f)$ is (topologically) weakly mixing if for any nonempty open subsets $U_{1}, U_{2}, V_{1}$, and $V_{2}$ of $X$, there exists an $n \in \mathbb{N}$ such that $f^{n}\left(U_{1}\right) \cap V_{1} \neq \emptyset$ and $f^{n}\left(U_{2}\right) \cap V_{2} \neq \emptyset$. It follows from these definitions that weak mixing implies transitivity.

In [5], Blanchard introduced overall properties and partial properties. For example, sensitive dependence on initial conditions, Devaney chaos (see [6]), weak mixing, mixing, and more belong to overall properties; Li-Yorke chaos (see $[7])$ and positive entropy (see $[1,8]$ ) belong to partial properties. Weak mixing is an overall property; it is stable under semiconjugate maps and implies Li-Yorke chaos. We have a weakly mixing system that always contains a dense uncountable scrambled set (see [9]). In [10], Blanchard and Huang introduced the concepts of weakly mixing subset, derived from a result given by Xiong and Yang [11] and showed "partial weak mixing implies Li-Yorke chaos" and "Li-Yorke chaos can not imply partial weak mixing."

Motivated by the idea of Blanchard and Huang's notion of "weakly mixing subset," Oprocha and Zhang [12] extended the notion of weakly mixing subset, gave the concept of "transitive subset," and discussed its basic properties. In recent years, many authors studied the dynamical properties for set-valued discrete systems. Román-Flores [13], Banks [14], Peris [15], Wang and Wei [16], and Acosta et al. [17] investigated the properties of topological transitivity and weak mixing for set-valued discrete systems. Fedeli [18], Guirao et al. [19] and Hou et al. [20] studied Devaney chaos for set-valued discrete systems. Lampart and Raith [21] discussed topological entropy for set-valued maps. Liu et al. [22] and Wang et al. [23] studied sensitivity of set-valued discrete systems. Wu and Xue [24] discussed shadowing property for induced setvalued dynamical systems. Also, we continue to discuss transitive subsets, weakly mixing subsets for set-valued discrete systems, and investigate the relationship between set-valued discrete system and original system on transitive subset, weakly mixing subset. More precisely, a set-valued discrete system has a transitive subset if and only if original system has a weakly mixing subset and we give an example showing that 
original system has a transitive subset which does not imply set-valued discrete system has a transitive subset. Moreover, we prove that a transitive point of set-valued discrete system is a transitive subset of original system.

\section{Preliminaries}

A TDS $(X, f)$ is point transitive if there exists a point $x_{0} \in$ $X$ with dense orbit, that is, $\overline{\operatorname{orb}\left(x_{0}\right)}=X$, where $\overline{\operatorname{orb}\left(x_{0}\right)}$ denotes the closure of $\operatorname{orb}\left(x_{0}\right)$. Such a point $x_{0}$ is called transitive point of $(X, f)$. If $X$ is a compact metric space without isolated points, then topologically transitive and point transitive are equivalent (see [2]). A TDS $(X, f)$ is minimal if $\overline{\operatorname{orb}(x, f)}=X$ for every $x \in X$; that is, every point is transitive point. A point $x$ is called minimal if the subsystem $(\overline{\operatorname{orb}(x, f)}, f)$ is minimal.

The distance from a point $x$ to a nonempty set $A$ in $X$ is defined by

$$
d(x, A)=\inf _{a \in A} d(x, a)
$$

Let $2^{X}$ be the family of all nonempty compact subsets of $X$. The Hausdorff metric on $2^{X}$ is defined by

$$
\begin{array}{r}
d_{H}(A, B)=\max \left\{\sup _{a \in A} d(a, B), \sup _{b \in B} d(b, A)\right\} \\
\text { for every } A, B \in 2^{X} .
\end{array}
$$

It follows from Michael [25] and Engelking [26] that $2^{X}$ is a compact metric space. The Vietoris topology $\tau_{v}$ on $2^{X}$ is generated by the base

$$
\begin{aligned}
& v\left(U_{1}, U_{2}, \ldots, U_{n}\right)=\left\{F \in 2^{X}: F \subseteq \bigcup_{i=1}^{n} U_{i},\right. \\
& \left.F \cap U_{i} \neq \emptyset \forall i \leq n\right\},
\end{aligned}
$$

where $U_{1}, U_{2}, \ldots, U_{n}$ are open subsets of $X$.

Let $2^{f}$ be the induced set-valued map defined by

$$
2^{f}: 2^{X} \longrightarrow 2^{X}, \quad 2^{f}(F)=f(F) \quad \text { for every } F \in 2^{X} \text {. }
$$

Then $2^{f}$ is well defined. $\left(2^{X}, 2^{f}\right)$ is called a set-valued discrete system.

Let $X$ be $T_{1}$ space; that is, single point set is closed. Then $2^{A}=\left\{F \in 2^{X}: F \subseteq A\right\}$ is a closed subset of $2^{X}$ for any nonempty closed subset $A$ of $X$ (see [25]).

Definition 1 (see [10]). Let $(X, f)$ be a TDS and let $A$ be a closed subset of $X$ with at least two elements. $A$ is said to be weakly mixing if for any $k \in \mathbb{N}$, any choice of nonempty open subsets $V_{1}, V_{2}, \ldots, V_{k}$ of $A$ and nonempty open subsets $U_{1}, U_{2}, \ldots, U_{k}$ of $X$ with $A \cap U_{i} \neq \emptyset, i=1,2, \ldots, k$, there exists an $m \in \mathbb{N}$ such that $f^{m}\left(V_{i}\right) \cap U_{i} \neq \emptyset$ for $1 \leq i \leq k$. $(X, f)$ is called partial weak mixing if $X$ contains a weakly mixing subset.
Definition 2 (see [12]). Let $(X, f)$ be a TDS and $A$ be a nonempty subset of $X$. $A$ is called a transitive subset of $(X, f)$ if for any choice of nonempty open subset $V^{A}$ of $A$ and nonempty open subset $U$ of $X$ with $A \cap U \neq \emptyset$, there exists an $n \in \mathbb{N}$ such that $f^{n}\left(V^{A}\right) \cap U \neq \emptyset$.

Remark 3. (1) $(X, f)$ is topologically transitive if and only if $X$ is a transitive subset of $(X, f)$.

(2) By [12], $A$ is a transitive subset if and only if $\bar{A}$ is a transitive subset, where $\bar{A}$ denotes the closure of $A$.

According to the definitions of transitive subset and weakly mixing subset, we have the following.

Result 1. If $A$ is a weakly mixing subset of $(X, f)$, then $A$ is a transitive subset of $(X, f)$.

Result 2. If $a \in X$ is a transitive point of $(X, f)$, then $\{a\}$ is a transitive subset of $(X, f)$.

Result 3. If $A=\operatorname{orb}(x, f)$ is a periodic orbit of $(X, f)$ for some $x \in X$, then $A$ is a transitive subset of $(X, f)$.

Example 4. Tent map

$$
f(x)= \begin{cases}2 x, & \text { if } 0 \leq x \leq \frac{1}{2}, \\ 2(1-x), & \text { if } \frac{1}{2} \leq x \leq 1\end{cases}
$$

is shown in Figures 1 and 2, which is known to be transitive on $I=[0,1]$ (see $[6])$. We prove that $[1 / 4,3 / 4]$ is a transitive subset of $(X, f)$.

Let $S\left(f^{k}\right)$ denote the set of extreme value points of $f^{k}$ for every $k \in \mathbb{N}$. Then $S\left(f^{k}\right)=\left\{1 / 2^{k}, 2 / 2^{k}, \ldots,\left(2^{k}-1\right) / 2^{k}\right\}$. Since $S(f)=\{1 / 2\}, f(1 / 2)=1, f(0)=0$, and $f(1)=0$, we have

$$
f^{k}(x)= \begin{cases}1, & \text { if } x=\frac{1}{2^{k}}, \frac{3}{2^{k}}, \ldots, \frac{2^{k}-1}{2^{k}} \\ 0, & \text { if } x=0, \frac{2}{2^{k}}, \frac{4}{2^{k}}, \ldots, \frac{2^{k}-2}{2^{k}}, 1 .\end{cases}
$$

Let $I_{k}^{j}=\left[j / 2^{k},(j+1) / 2^{k}\right]$ for $0 \leq j \leq 2^{k}-1$. Then $f^{k}\left(I_{k}^{j}\right)=$ $[0,1]$. For any nonempty open set $U$ of $[1 / 4,3 / 4]$, without loss of generality, we take $U=\left(x_{0}-\varepsilon, x_{0}+\varepsilon\right)$ for a given $\varepsilon>0$ and $x_{0} \in \operatorname{int}[1 / 4,3 / 4]$, where int $[1 / 4,3 / 4]$ denotes the interior of $[1 / 4,3 / 4]$. When $l \in \mathbb{N}$ and $l>\log _{2}(1 / \varepsilon)$, then there exist $j \in \mathbb{Z}_{+}$and $0 \leq j \leq 2^{l}-1$ such that $I_{l}^{j} \subseteq U$. Furthermore, we have $f^{l}(U)=[0,1]$. Thus, for any nonempty open set $U$ of $[1 / 4,3 / 4]$ and nonempty open set $V$ of $[0,1]$ with $V \cap$ $[1 / 4,3 / 4] \neq \emptyset$, there exists a $k \in \mathbb{N}$ such that $f^{k}(U) \cap V \neq \emptyset$. This shows that $[1 / 4,3 / 4]$ is a transitive subset of $(I, f)$.

Definition 5 (see [27]). Let $(X, \tau)$ be a topological space and $A$ be a nonempty set of $X$. $A$ is a regular closed set of $X$ if $A=\overline{\operatorname{int}(A)}$, where $\operatorname{int}(A)$ denotes the interior of $A$. 


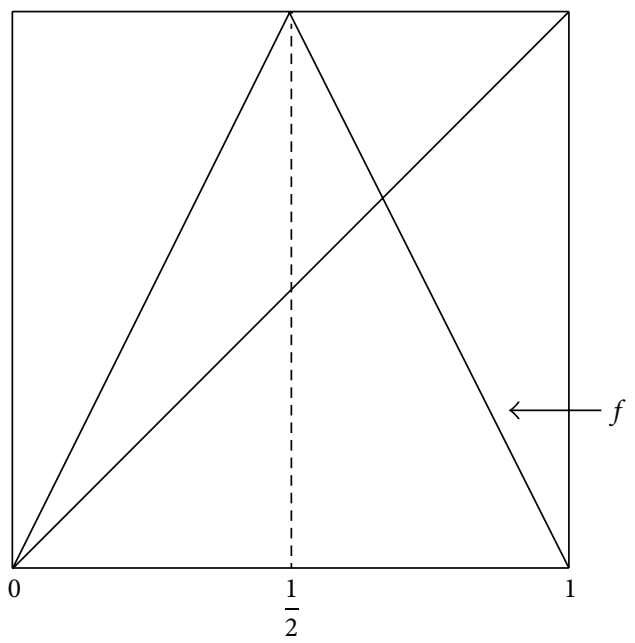

FIGURE 1



FigURE 2

We easily prove that $A$ is a regular closed set if and only if $\operatorname{int}\left(V^{A}\right) \neq \emptyset$ for any nonempty set $V^{A}$ of $A$.

Theorem 6 (see $[14,15]$ ). Let $X$ be a compact space, and let $2^{X}$ be equipped with the Vietoris topology. If $f: X \rightarrow X$ is a continuous map, then $2^{f}: 2^{X} \rightarrow 2^{X}$ is continuous and $(X, f)$ is weakly mixing $\Leftrightarrow\left(2^{X}, 2^{f}\right)$ is weakly mixing $\Leftrightarrow\left(2^{X}, 2^{f}\right)$ is topologically transitive.

\section{Transitive Subsets and Weakly Mixing Subsets of Set-Valued Discrete Systems}

For a $\operatorname{TDS}(X, f)$ and two nonempty subsets $U, V \subseteq X$, we use the following notation:

$$
N(U, V)=\left\{n \in \mathbb{N}: f^{n}(U) \cap V \neq \emptyset\right\} .
$$

Theorem 7. $A$ is a weakly mixing subset of $(X, f)$ if and only if $2^{A}$ is a weakly mixing subset of $\left(2^{X}, 2^{f}\right)$.
Proof

Necessity. We prove for any $k \in \mathbb{N}$, any choice of nonempty open subsets $\mathscr{V}_{1}^{2^{A}}, \mathscr{V}_{2}^{2^{A}}, \ldots, \mathscr{V}_{k}^{2^{A}}$ of $2^{A}$ and nonempty open subsets $\mathscr{U}_{1}, \mathscr{U}_{2}, \ldots, \mathscr{U}_{k}$ of $2^{X}$ with $2^{A} \cap \mathscr{U}_{i} \neq \emptyset$ for $i=$ $1,2, \ldots, k$, that there exists an $m \in \mathbb{N}$ such that

$$
\left(2^{f}\right)^{m}\left(\mathscr{V}_{i}^{2^{A}}\right) \cap \mathscr{U}_{i} \neq \emptyset \quad \text { for } i=1,2, \ldots, k
$$

For nonempty open subset $\mathscr{V}_{i}^{2^{A}}$ of $2^{A}$, there exist open subsets $\mathscr{V}_{i}$ of $2^{X}$ such that $\mathscr{V}_{i}^{2^{A}}=\mathscr{V}_{i} \cap 2^{A}$ for $i=1,2, \ldots, k$. Without loss of generality, let

$$
\begin{array}{r}
\mathscr{V}_{i}=v\left(V_{1}^{i}, V_{2}^{i}, \ldots, V_{n}^{i}\right), \quad \mathscr{U}_{i}=v\left(U_{1}^{i}, U_{2}^{i}, \ldots, U_{n}^{i}\right) \\
\text { for } i=1,2, \ldots, k .
\end{array}
$$

$V_{j}^{i}$ and $U_{j}^{i}$ are nonempty open subsets of $X$ for $i=1,2, \ldots$, $k, j=1,2, \ldots, n$. Furthermore,

$$
\begin{aligned}
\mathscr{V}_{i}^{2^{A}}=\{ & \left\{F \in 2^{A}: F \subseteq \bigcup_{j=1}^{n}\left(V_{j}^{i} \cap A\right),\right. \\
& \left.F \cap\left(V_{j}^{i} \cap A\right) \neq \emptyset \forall 1 \leq j \leq n\right\} .
\end{aligned}
$$

Let $\left(V_{j}^{i}\right)^{A}=V_{j}^{i} \cap A$. Then we have $\left(V_{j}^{i}\right)^{A} \neq \emptyset$ for $j=1,2, \ldots, n$. Moreover, $\mathcal{U}_{i} \cap 2^{A} \neq \emptyset$, then $U_{j}^{i} \cap A \neq \emptyset$ for $j=1,2, \ldots, n$.

We consider any nonempty open subsets $\left(V_{1}^{1}\right)^{A}, \ldots$, $\left(V_{n}^{1}\right)^{A}, \ldots,\left(V_{1}^{k}\right)^{A}, \ldots,\left(V_{n}^{k}\right)^{A}$ of $A$ and any nonempty open subsets $U_{1}^{1}, \ldots, U_{n}^{1}, \ldots, U_{1}^{k}, \ldots, U_{n}^{k}$ of $X$ with $A \cap U_{j}^{i} \neq \emptyset$ for $i=$ $1,2, \ldots, k, j=1,2, \ldots, n$. Since $A$ is a weakly mixing subset of $(X, f)$, then there exists an $m \in \mathbb{N}$ such that

$$
\begin{array}{r}
\left(V_{j}^{i}\right)^{A} \cap f^{-m}\left(U_{j}^{i}\right) \neq \emptyset \quad \text { for } i=1,2, \ldots, k, \\
j=1,2, \ldots, n .
\end{array}
$$

Take $x_{j}^{i} \in\left(V_{j}^{i}\right)^{A} \cap f^{-m}\left(U_{j}^{i}\right)$ for $i=1,2, \ldots, k, j=1,2, \ldots, n$. We have $x_{j}^{i} \in V_{j}^{i} \cap A$ and $f^{m}\left(x_{j}^{i}\right) \in U_{j}^{i}$ for $i=1,2, \ldots, k, j=$ $1,2, \ldots, n$. Let $B_{i}=\bigcup_{j=1}^{n}\left\{x_{j}^{i}\right\}$. Then $B_{i} \in \mathscr{V}_{i}^{2^{A}}$ and $f^{m}\left(B_{i}\right) \in$ $\mathcal{U}_{i}$. Furthermore, we have $f^{m}\left(B_{i}\right) \in f^{m}\left(\mathscr{V}_{i}^{2^{A}}\right)$. Therefore, $\left(2^{f}\right)^{m}\left(\mathscr{V}_{i}^{2^{A}}\right) \cap \mathcal{U}_{i} \neq \emptyset$ for $i=1,2, \ldots, k$.

Sufficiency. We show that for any $k \in \mathbb{N}$, any choice of nonempty open subsets $V_{1}^{A}, V_{2}^{A}, \ldots, V_{k}^{A}$ of $A$ and nonempty open subsets $U_{1}, U_{2}, \ldots, U_{k}$ of $X$ with $A \cap U_{i} \neq \emptyset$ for each $i=1,2, \ldots, k$, there exists an $m \in \mathbb{N}$ such that $f^{m}\left(V_{i}^{A}\right) \cap U_{i} \neq \emptyset$ for $i=1,2, \ldots, k$.

For nonempty open subset $V_{i}^{A}$ for $i=1,2, \ldots, k$, there exists an open subset $V_{i}$ of $X$ such that $V_{i}^{A}=V_{i} \cap A$ for $i=$ $1,2, \ldots, k$. Let

$$
\mathscr{V}_{i}^{2^{A}}=v\left(V_{i}\right) \cap 2^{A}, \quad \mathcal{U}_{i}=v\left(U_{i}\right) \quad \text { for } i=1,2, \ldots, k .
$$


Then $\mathscr{V}_{i}^{2^{A}}$ is a nonempty open subset of $2^{A}$ and $\mathcal{U}_{i}$ is a nonempty open set of $2^{X}$ with $2^{A} \cap \mathscr{U}_{i} \neq \emptyset$ for $i=1,2, \ldots, k$. Since $2^{A}$ is a weakly mixing subset of $\left(2^{X}, 2^{f}\right)$, there exists an $m \in \mathbb{N}$ such that

$$
\mathscr{V}_{i}^{2^{A}} \cap\left(2^{f}\right)^{-m}\left(\mathcal{U}_{i}\right) \neq \emptyset \quad \text { for } i=1,2, \ldots, k .
$$

Take $F_{i} \in \mathscr{V}_{i}^{2^{A}} \cap\left(2^{f}\right)^{-m}\left(\mathscr{U}_{i}\right)$. We have $F_{i} \in \nu\left(V_{i}\right) \cap 2^{A}$ and $F_{i} \in\left(2^{f}\right)^{-m}\left(\mathcal{U}_{i}\right)$ for $i=1,2, \ldots, k$. Therefore, $F_{i} \subseteq\left(V_{i} \cap A\right) \cap$ $f^{-m}\left(U_{i}\right)$. Furthermore, we have $V_{i}^{A} \cap f^{-m}\left(U_{i}\right) \neq \emptyset$ for $i=1,2$, $\ldots, k$. This shows $A$ is a weakly mixing subset of $(X, f)$.

Theorem 8. Let $A$ be a nonempty closed set of $X$. If $2^{A}$ is a transitive subset of $\left(2^{X}, 2^{f}\right)$, then $A$ is a transitive subset of $(X, f)$.

Proof. We show that for any choice of nonempty open subset $V^{A}$ of $A$ and nonempty open subset $U$ of $X$ with $A \cap U \neq \emptyset$, there exists an $n \in \mathbb{N}$ such that $f^{n}\left(V^{A}\right) \cap U \neq \emptyset$.

For nonempty open subset $V^{A}$ of $A$, there exists a nonempty open subset $V$ of $X$ such that $V^{A}=V \cap A$. Let $\mathcal{U}=$ $v(U), \mathscr{V}=v(V)$, and $\mathscr{V}^{2^{A}}=v(V) \cap 2^{A}$; then $\mathscr{V}^{2^{A}}$ is a nonempty open subset of $2^{A}$. Moreover, $U \cap A \neq \emptyset$ implies that $\nu(U) \cap 2^{A} \neq \emptyset$. Since $2^{A}$ is a topologically transitive subset of $\left(2^{X}, 2^{f}\right)$, there exists an $n \in \mathbb{N}$ such that $\mathscr{V}^{2^{A}} \cap\left(2^{f}\right)^{-n}(\mathcal{U}) \neq \emptyset$. Furthermore, there exists $F \in \mathscr{V}^{2^{A}} \cap\left(2^{f}\right)^{-n}(\mathcal{U})$ such that $F \in$ $\mathscr{V}^{2^{A}}$ and $f^{n}(F) \in \mathcal{U}$, which implies $F \subseteq V^{A}$ and $f^{n}(F) \subseteq U$. Therefore, we have $f^{n}\left(V^{A}\right) \cap U \neq \emptyset$.

Lemma 9. Let $A$ be a regular closed set of $X$ but not a singleton. $A$ is a weakly mixing subset of $(X, f)$ if and only if for any choice of nonempty open subsets $V_{1}^{A}, V_{2}^{A}$ of $A$ and nonempty open subsets $U_{1}, U_{2}$ of $X$ with $A \cap U_{i} \neq \emptyset, i=1,2$, there exists an $n \in \mathbb{N}$ such that $f^{n}\left(V_{i}^{A}\right) \cap U_{i} \neq \emptyset$ for $i=1,2$.

Proof. Necessity is obvious by the definition of weakly mixing subset. We need only to prove sufficiency.

Let $V_{1}^{A}, V_{2}^{A}$ be two nonempty open subsets of $A$ and let $U_{1}, U_{2}$ be two nonempty open subsets of $X$ with $A \cap U_{i} \neq \emptyset$, $i=1,2$. Since $A$ is a regular closed set of $X$, then $\operatorname{int}\left(V_{2}^{A}\right) \neq \emptyset$. We consider two nonempty open subsets $V_{1}^{A}, U_{1} \cap A$ of $A$ and two nonempty open subsets $\operatorname{int}\left(V_{2}^{A}\right), U_{2}$ of $X$; there exists an $n \in \mathbb{N}$ such that $f^{n}\left(V_{1}^{A}\right) \cap \operatorname{int}\left(V_{2}^{A}\right) \neq \emptyset$ and $f^{n}\left(U_{1} \cap A\right) \cap U_{2} \neq \emptyset$. Furthermore, we have $V_{1}^{A} \cap f^{-n}\left(\operatorname{int}\left(V_{2}^{A}\right)\right) \neq \emptyset$ and $U_{1} \cap A \cap$ $f^{-n}\left(U_{2}\right) \neq \emptyset$.

Let $V=V_{1}^{A} \cap f^{-n}\left(\operatorname{int}\left(V_{2}^{A}\right)\right)$ and $U=U_{1} \cap f^{-n}\left(U_{2}\right) \neq \emptyset$. Then $V$ is a nonempty open subset of $A$ and $U$ is a nonempty open subset of $X$ with $A \cap U \neq \emptyset$. By assumption, $N(U, V) \neq \emptyset$. For any $m \in N(U, V)$, we have $f^{m}(V) \cap U \subseteq f^{m}\left(V_{1}^{A}\right) \cap U_{1}$, which implies $f^{m}\left(V_{1}^{A}\right) \cap U_{1} \neq \emptyset$. Since $f^{n}(V) \subseteq \operatorname{int}\left(V_{2}^{A}\right)$, $f^{n}(U) \subseteq U_{2}$, it follows that

$$
\begin{aligned}
f^{n}\left(f^{m}(V) \cap U\right) & \subseteq f^{m+n}(V) \cap f^{n}(U) \\
& \subseteq f^{m}\left(\operatorname{int}\left(V_{2}^{A}\right)\right) \cap f^{n}(U) \\
& \subseteq f^{m}\left(V_{2}^{A}\right) \cap U_{2} .
\end{aligned}
$$

Hence, $f^{m}\left(V_{2}^{A}\right) \cap U_{2} \neq \emptyset$. Furthermore, we have $m \in$ $N\left(V_{1}^{A}, U_{1}\right) \cap N\left(V_{2}^{A}, U_{2}\right)$ and $N(U, V) \subseteq N\left(V_{1}^{A}, U_{1}\right) \cap$ $N\left(V_{2}^{A}, U_{2}\right)$. This shows that for any $k \in \mathbb{N}$, any choice of nonempty open subsets $V_{1}^{A}, V_{2}^{A}, \ldots, V_{k}^{A}$ of $A$ and nonempty open subsets $U_{1}, U_{2}, \ldots, U_{k}$ of $X$ with $A \cap U_{i} \neq \emptyset$ for $i=$ $1,2, \ldots, k$, we have $\bigcap_{i=1}^{k} N\left(V_{i}^{A}, U_{i}\right) \neq \emptyset$. This means that there exists an $n \in \mathbb{N}$ such that $f^{n}\left(V_{i}^{A}\right) \cap U_{i} \neq \emptyset$ for $i=1,2, \ldots, k$. Therefore, $A$ is a weakly mixing subset of $(X, f)$.

Lemma 10. Let $A$ be a regular closed set of $X$ but not a singleton. A is a weakly mixing subset of $(X, f)$ if and only iffor any choice of nonempty open subset $V^{A}$ of $A$ and nonempty open subsets $U, W$ of $X$ with $A \cap U \neq \emptyset$ and $A \cap W \neq \emptyset$, there exists an $n \in \mathbb{N}$ such that $f^{n}\left(V^{A}\right) \cap U \neq \emptyset$ and $f^{n}\left(V^{A}\right) \cap W \neq \emptyset$.

Proof. Necessity is obviously by the definition of weakly mixing subset. We need only prove sufficiency.

By Lemma 9, we only prove that for any choice of nonempty open subsets $V_{1}^{A}, V_{2}^{A}$ of $A$ and nonempty open subsets $U_{1}, U_{2}$ of $X$ with $A \cap U_{i} \neq \emptyset, i=1,2$, there exists an $m \in \mathbb{N}$ such that $f^{m}\left(V_{i}^{A}\right) \cap U_{i} \neq \emptyset$ for $i=1,2$.

Let $V_{1}$ and $V_{2}$ be two open sets of $X$ satisfying $V_{1}^{A}=V_{1} \cap A$ and $V_{2}^{A}=V_{2} \cap A$. Since $A$ is a regular closed set, then $\operatorname{int}\left(V_{1}^{A}\right) \neq \emptyset$ and $\operatorname{int}\left(V_{2}^{A}\right) \neq \emptyset$. We consider nonempty open subset $V_{1}^{A}$ of $A$ and nonempty open subsets $\operatorname{int}\left(V_{2}^{A}\right), U_{2}$ of $X$, according to the assumption that there exists an $n \in \mathbb{N}$ such that

$$
\begin{gathered}
P^{A}=V_{1}^{A} \cap f^{-n}\left(\operatorname{int}\left(V_{2}^{A}\right)\right) \neq \emptyset, \\
Q^{A}=V_{1}^{A} \cap f^{-n}\left(U_{2}\right) \neq \emptyset .
\end{gathered}
$$

Moreover, $P=V_{1} \cap f^{-n}\left(\operatorname{int}\left(V_{2}^{A}\right)\right)$ and $Q=V_{1} \cap f^{-n}\left(U_{2}\right)$ are nonempty open sets of $X$ with $P \cap A \neq \emptyset$ and $Q \cap A \neq \emptyset$. We consider nonempty open subset $P^{A}$ of $A$ and nonempty open subsets $Q, U_{1}$ of $X$; there exists an $m \in \mathbb{N}$ such that $f^{m}\left(P^{A}\right) \cap Q \neq \emptyset$ and $f^{m}\left(P^{A}\right) \cap U_{1} \neq \emptyset$. As $f^{m}\left(P^{A}\right) \cap U_{1} \subseteq$ $f^{m}\left(V_{1}^{A}\right) \cap U_{1}$, we have $f^{m}\left(V_{1}^{A}\right) \cap U_{1} \neq \emptyset$. Since $f^{m}\left(P^{A}\right) \cap Q \neq \emptyset$, then $f^{m}\left(f^{-n}\left(V_{2}^{A}\right)\right) \cap f^{-n}\left(U_{2}\right) \neq \emptyset$, which implies $f^{m}\left(V_{2}^{A}\right) \cap$ $U_{2} \neq \emptyset$. Therefore, by Lemma $9, A$ is a weakly mixing subset of $(X, f)$.

Theorem 11. Let $A$ be a regular closed subset of $X$ but not a singleton. If $2^{A}$ is a transitive subset of $\left(2^{X}, 2^{f}\right)$, then $A$ is a weakly mixing subset of $(X, f)$.

Proof. Suppose $A$ is a regular closed subset of $X$ but not a singleton. Then $2^{A}$ is a closed subset of $2^{X}$ but not a singleton. Let $V^{A}$ is a nonempty open subset of $A$, and let $U$ and $W$ be two nonempty open subsets of $X$ with $A \cap U \neq \emptyset$ and $A \cap$ $W \neq \emptyset$. According to Lemma 10, we only prove there exists an $n \in \mathbb{N}$ such that $f^{n}\left(V^{A}\right) \cap U \neq \emptyset$ and $f^{n}\left(V^{A}\right) \cap W \neq \emptyset$.

For nonempty open subset $V^{A}$ of $A$, there exists an open subset $V$ of $X$ such that $V^{A}=V \cap A$. Let $\mathscr{U}=\nu(V)$ and $\mathscr{V}=$ $v(U, W)$, then $\mathscr{U}$ and $\mathscr{V}$ are open subsets of $2^{X}$ with $2^{A} \cap \mathcal{U} \neq \emptyset$ and $2^{A} \cap \mathscr{V} \neq \emptyset$. We consider nonempty open subset $\mathscr{U} \cap 2^{A}$ of 
$2^{A}$ and nonempty open subset $\mathscr{V}$ of $2^{X}$. Since $2^{A}$ is a transitive subset of $\left(2^{X}, 2^{f}\right)$, there exists an $n \in \mathbb{N}$ such that

$$
\left(\mathscr{U} \cap 2^{A}\right) \cap\left(2^{f}\right)^{-n}(\mathscr{V}) \neq \emptyset .
$$

Take $B \in\left(\mathscr{U} \cap 2^{A}\right) \cap\left(2^{f}\right)^{-n}(\mathscr{V})$. We have $B \subseteq V^{A},\left(2^{f}\right)^{n}(B) \cap$ $U \neq \emptyset$, and $\left(2^{f}\right)^{n}(B) \cap W \neq \emptyset$, that is, $B \subseteq V^{A}, f^{n}(B) \cap U \neq \emptyset$, and $f^{n}(B) \cap W \neq \emptyset$, which implies $f^{n}\left(V^{A}\right) \cap U \neq \emptyset$ and $f^{n}\left(V^{A}\right) \cap$ $W \neq \emptyset$. This shows $A$ is a weakly mixing subset of $(X, f)$.

By Theorems 7 and 11, we have the following corollary.

Corollary 12. Let $A$ be a regular closed subset of $X$ but not a singleton. Then the following properties are equivalent:

(1) $A$ is a weakly mixing subset of $(X, f)$;

(2) $2^{A}$ is a weakly mixing subset of $\left(2^{X}, 2^{f}\right)$;

(3) $2^{A}$ is a transitive subset of $\left(2^{X}, 2^{f}\right)$.

Lemma 13. Let $A$ be a transitive point of $\left(2^{X}, 2^{f}\right)$. Then $x$ is a transitive point of $(X, f)$ for every $x \in A$.

Proof. Suppose that $A$ is a transitive point of $\left(2^{X}, 2^{f}\right)$. Then for any open $\operatorname{set} v\left(U_{1}, U_{2}, \ldots, U_{m}\right)$ of $\left(2^{X}, 2^{f}\right)$, there exists $k \epsilon$ $\mathbb{Z}_{+}$such that

$$
\left(2^{f}\right)^{k}(A) \in v\left(U_{1}, U_{2}, \ldots, U_{m}\right) .
$$

In particular, take $U_{1}=U_{2}=\cdots=U_{m}=U$; there exists $l \in \mathbb{Z}_{+}$such that $\left(2^{f}\right)^{l}(A) \in v(U)$. Furthermore, for any $x \in A$, we have $f^{l}(x) \in U$. Since $U$ is any nonempty open set of $X$, it follows that $x$ is a transitive point of $(X, f)$.

Theorem 14. Let $A$ be a transitive point of $\left(2^{X}, 2^{f}\right)$. Then $A$ is a transitive subset of $(X, f)$.

Proof. Suppose that $A$ is a transitive point of $\left(2^{X}, 2^{f}\right)$. Then $A$ is a nonempty closed set of $X$. Let $V^{A}$ be a nonempty open set of $A$ and let $U$ be a nonempty open set of $X$ with $A \cap U \neq \emptyset$. We prove that there exists an $n \in \mathbb{N}$ such that $f^{n}\left(V^{A}\right) \cap U \neq \emptyset$.

Since $A$ is a transitive point of $\left(2^{X}, 2^{f}\right)$, by Lemma $13, x$ is a transitive point of $(X, f)$ for every $x \in A$. Let $V^{A}=V \cap A$, where $V$ is an open set of $X$. Let $x \in V^{A}$. Then $\overline{\operatorname{orb}(x, f)}=$ $X$. It means that there exists an $n \in \mathbb{N}$ such that $f^{n}(x) \in$ $U$. Furthermore, we have $f^{n}\left(V^{A}\right) \cap U \neq \emptyset$. Therefore, $A$ is a transitive subset of $(X, f)$.

Example 15. Let $S^{1}$ be the unit circle and let $T_{\lambda}: S^{1} \rightarrow S^{1}$ be a translation map such that

$$
T_{\lambda}(\theta)=\theta+2 \lambda \pi, \quad \lambda \in \mathbb{R} .
$$

If $\lambda$ is an irrational number, then $A=[0, \pi]$ is a transitive subset of $\left(S^{1}, T_{\lambda}\right)$, but $2^{A}$ is not a transitive subset of $\left(2^{S^{1}}, 2^{T}\right)$.

It is well known that if $\lambda=q / p$ is a rational number, then all points are periodic of period $q$, and so the set of periodic

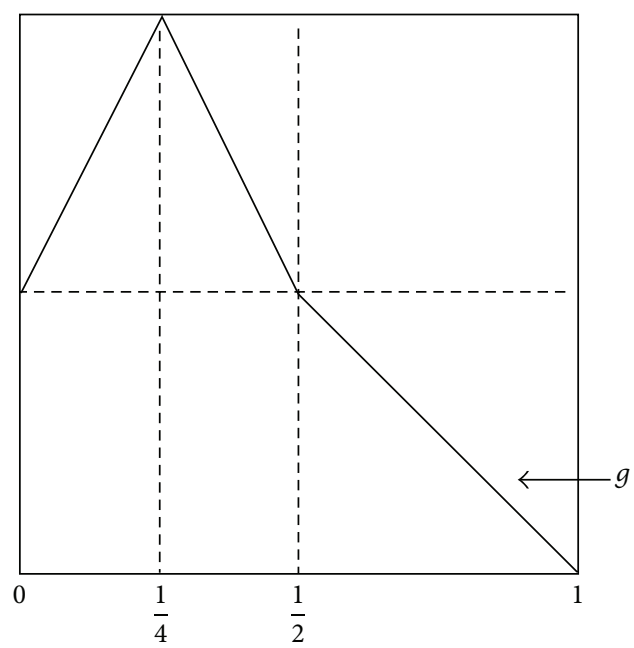

Figure 3

points is, obviously, dense in $S^{1}$. Moreover, by Jacobi's Theorem [6], if $\lambda$ is an irrational number, then each orbit $\left\{T_{\lambda}^{n}(\theta)\right.$ : $n \in \mathbb{N}\}$ is dense in $S^{1}$. Since $S^{1}$ is a compact metric space. Hence, $\left(S^{1}, T_{\lambda}\right)$ is topologically transitive.

Let $A=[0, \pi]$. Then $A$ is a nonempty closed subset of $S^{1}$. Let $V^{A}$ be a nonempty subset of $A$ and $U$ is an open subset of $S^{1}$ with $A \cap U \neq \emptyset$. Take $x \in V^{A}$. Since $\left\{T_{\lambda}^{n}(x): n \in \mathbb{N}\right\}$ is dense in $S^{1}$, there exists an $m \in \mathbb{N}$ such that $T_{\lambda}^{m}(x) \in U$. Furthermore, we have $T_{\lambda}^{m}\left(V^{A}\right) \cap U \neq \emptyset$. Therefore, $A$ is a transitive subset of $\left(S^{1}, T_{\lambda}\right)$; that is, $[0, \pi]$ is a transitive subset of $\left(S^{1}, T_{\lambda}\right)$.

Let $K=[0,1] \in 2^{A}$. Then $\operatorname{diam}(K)=\operatorname{diam}\left(2^{T_{\lambda}}(K)\right)=1$, where $\operatorname{diam}(K)$ denotes the diameter of $K$. Put $\varepsilon>0$ such that $1-\varepsilon>\varepsilon$. Let $\mathscr{V}=B(K, \varepsilon / 2)$ and $\mathscr{U}=B(\{1\}, \varepsilon / 2)$. Then $\mathscr{V}^{2^{A}}=$ $\mathscr{V} \cap 2^{A}$ is a nonempty open subset of $2^{A}$ and $\mathscr{U}$ is an open subset of $S^{1}$ with $2^{A} \cap \mathscr{U} \neq \emptyset$. Moreover, for any $F \in \mathscr{V}^{2^{A}}$ and any $G \in \mathcal{U}$, we have $\operatorname{diam}(F) \geq 1-\varepsilon$ and $\operatorname{diam}(G) \leq \varepsilon$. Furthermore, $\operatorname{diam}\left(\left(2^{T_{\lambda}}\right)^{n}(F)\right) \geq 1-\varepsilon>\varepsilon$ for all $n \in \mathbb{N}$. Therefore, $\left(2^{T_{\lambda}}\right)^{n}\left(\mathscr{V}^{A}\right) \cap \mathcal{U}=\emptyset$ for all $n \in \mathbb{N}$. It means that $2^{A}$ is not a transitive subset of $\left(2^{S^{1}}, 2^{T_{\lambda}}\right)$.

Example 16. Let $I=[0,1]$. Define $g: I \rightarrow I$ by

$$
g(x)= \begin{cases}\frac{1}{2}+2 x, & \text { if } 0 \leq x \leq \frac{1}{4}, \\ \frac{3}{2}-2 x, & \text { if } \frac{1}{4} \leq x \leq \frac{1}{2}, \\ 1-x, & \text { if } \frac{1}{2} \leq x \leq 1 .\end{cases}
$$

Then $\left(2^{I}, 2^{g}\right)$ has a weakly mixing subset (Figures 3 and 4 ).

Let $J=[0,1 / 2]$ and $K=[1 / 2,1]$. Then $g(J)=K$ and $g(K)=J$. Hence, $\left.g^{2}\right|_{K}$ is equal to the tent map $f$ of Example 4. Furthermore, by [8], $\left(K, g^{2}\right)$ is mixing. Hence, $K$ is a weakly mixing subset of $\left(K, g^{2}\right)$. We prove that $K$ is a weakly mixing subset of $(I, g)$.

For any $m \in \mathbb{N}$, any choice of nonempty open subsets $V_{1}^{K}, \ldots, V_{m}^{K}$ of $K$ and nonempty open subsets $U_{1}, \ldots, U_{m}$ of 


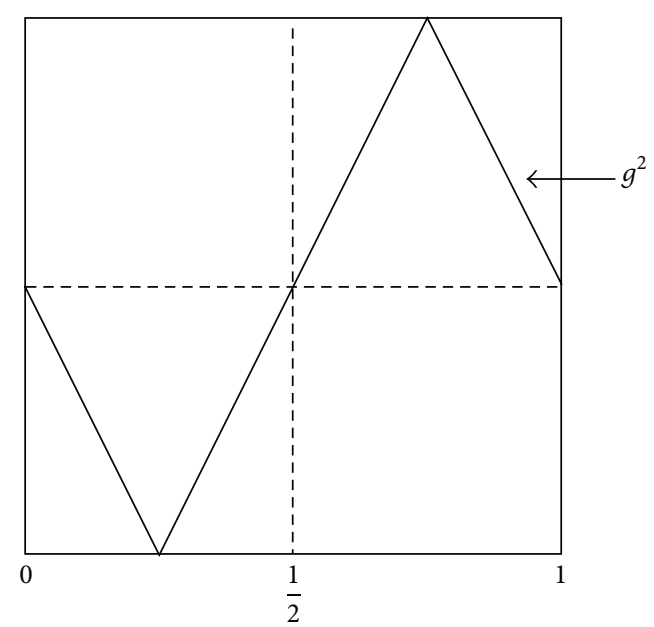

FIGURE 4

$I$ with $K \cap U_{i} \neq \emptyset, i=1,2, \ldots, m$, we have $K \cap U_{i}$ are nonempty open subsets of $K$ for all $i=1,2, \ldots, m$. Since $\left(K, g^{2}\right)$ is weak mixing, by [28], there exists an $n \in \mathbb{N}$ such that $\left(g^{2}\right)^{n}\left(V_{i}^{K}\right) \cap\left(K \cap U_{i}\right)=g^{2 n}\left(V_{i}^{K}\right) \cap\left(K \cap U_{i}\right) \neq \emptyset$ for $i=$ $1,2, \ldots, m$. Furthermore, we have $g^{2 n}\left(V_{i}^{K}\right) \cap U_{i} \neq \emptyset$ for $i=$ $1,2, \ldots, m$. Hence, $K$ is a weak mixing subset of $(I, g)$. By Theorem $7,2^{K}$ is a weakly mixing subset of $\left(2^{I}, 2^{g}\right)$.

\section{Acknowledgments}

The author would like to thank the referees for many valuable and constructive comments and suggestions for improving this paper. This work is supported by the Natural Science Foundation of Henan Province (122300410427), China.

\section{References}

[1] L. S. Block and W. A. Coppel, Dynamics in One Dimension, Springer, Berlin, Germany, 1992.

[2] S. Kolyada and L. Snoha, "Some aspects of topological transitivity-a survey," Grazer Mathematische Berichte, vol. 334, pp. 335, 1997.

[3] C. Robinson, Dynamical Systems, CRC Press, Boca Raton, Fla, USA, 1999.

[4] P. Walters, An Introduction to Ergodic Theory, vol. 79 of Graduate Texts in Mathematics, Springer-Verlag, New York, NY, USA, 1982.

[5] F. Blanchard, “Topological chaos: what may this mean?" Journal of Difference Equations and Applications, vol. 15, no. 1, pp. 23-46, 2009.

[6] R. L. Devaney, An Introduction to Chaotic Dynamical Systems, Addison-Wesley, Redwood City, Calif, USA, 2nd edition, 1989.

[7] T. Y. Li and J. A. Yorke, "Period three implies chaos," The American Mathematical Monthly, vol. 82, no. 10, pp. 985-992, 1975.

[8] S. Ruette, "Chaos for continuous interval maps: a survey of relationship between the various sorts of chaos," http://www .math.u-psud.fr/ ruette/.

[9] A. Iwanik, "Independence and scrambled sets for chaotic mappings," in The Mathematical Heritage of C.F. Gauss, pp. 372-378, World Scientific, River Edge, NJ, USA, 1991.
[10] F. Blanchard and W. Huang, "Entropy sets, weakly mixing sets and entropy capacity," Discrete and Continuous Dynamical Systems A, vol. 20, no. 2, pp. 275-311, 2008.

[11] J. C. Xiong and Z. G. Yang, "Chaos caused by a topologically mixing map," in Dynamical Systems and Related Topics, vol. 9, pp. 550-572, World Scientific, Singapore, 1991.

[12] P. Oprocha and G. Zhang, "On local aspects of topological weak mixing in dimension one and beyond," Studia Mathematica, vol. 202, no. 3, pp. 261-288, 2011.

[13] H. Román-Flores, "A note on transitivity in set-valued discrete systems," Chaos, Solitons and Fractals, vol. 17, no. 1, pp. 99-104, 2003.

[14] J. Banks, "Chaos for induced hyperspace maps," Chaos, Solitons and Fractals, vol. 25, no. 3, pp. 681-685, 2005.

[15] A. Peris, "Set-valued discrete chaos," Chaos, Solitons and Fractals, vol. 26, no. 1, pp. 19-23, 2005.

[16] Y. Wang and G. Wei, "Characterizing mixing, weak mixing and transitivity of induced hyperspace dynamical systems," Topology and its Applications, vol. 155, no. 1, pp. 56-68, 2007.

[17] G. Acosta, A. Illanes, and H. Méndez-Lango, "The transitivity of induced maps," Topology and Its Applications, vol. 156, no. 5, pp. 1013-1033, 2009.

[18] A. Fedeli, "On chaotic set-valued discrete dynamical systems," Chaos, Solitons and Fractals, vol. 23, no. 4, pp. 1381-1384, 2005.

[19] J. L. G. Guirao, D. Kwietniak, M. Lampart, P. Oprocha, and A. Peris, "Chaos on hyperspaces," Nonlinear Analysis. Theory, Methods \& Applications, vol. 71, no. 1-2, pp. 1-8, 2009.

[20] B. Hou, X. Ma, and G. Liao, "Difference between Devaney chaos associated with two systems," Nonlinear Analysis. Theory, Methods \& Applications, vol. 72, no. 3-4, pp. 1616-1620, 2010.

[21] M. Lampart and P. Raith, "Topological entropy for set valued maps," Nonlinear Analysis. Theory, Methods \& Applications, vol. 73, no. 6, pp. 1533-1537, 2010.

[22] H. Liu, E. Shi, and G. Liao, "Sensitivity of set-valued discrete systems," Nonlinear Analysis. Theory, Methods \& Applications, vol. 82, pp. 985-992, 2009.

[23] Y. Wang, G. Wei, and W. H. Campbell, "Sensitive dependence on initial conditions between dynamical systems and their induced hyperspace dynamical systems," Topology and its Applications, vol. 156, no. 4, pp. 803-811, 2009.

[24] Y. Wu and X. Xue, "Shadowing property for induced set-valued dynamical systems of some expansive maps," Dynamic Systems and Applications, vol. 19, no. 3-4, pp. 405-414, 2010.

[25] E. Michael, “Topologies on spaces of subsets," Transactions of the American Mathematical Society, vol. 71, pp. 152-182, 1951.

[26] R. Engelking, General Topology, PWN, Warsaw, Poland, 1977.

[27] R. F. Dickman Jr., "Regular closed maps," Proceedings of the American Mathematical Society, vol. 39, pp. 414-416, 1973.

[28] H. Furstenberg, "Disjointness in ergodic theory, minimal sets, and a problem in Diophantine approximation," Mathematical Systems Theory, vol. 1, pp. 1-49, 1967. 


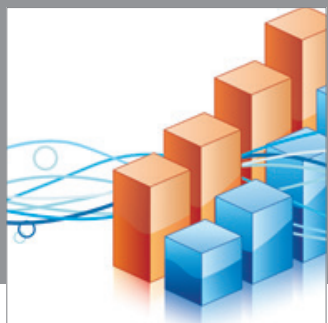

Advances in

Operations Research

mansans

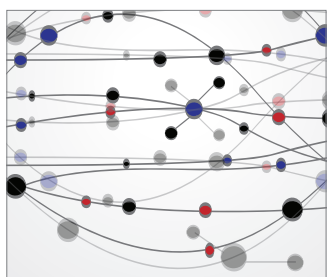

The Scientific World Journal
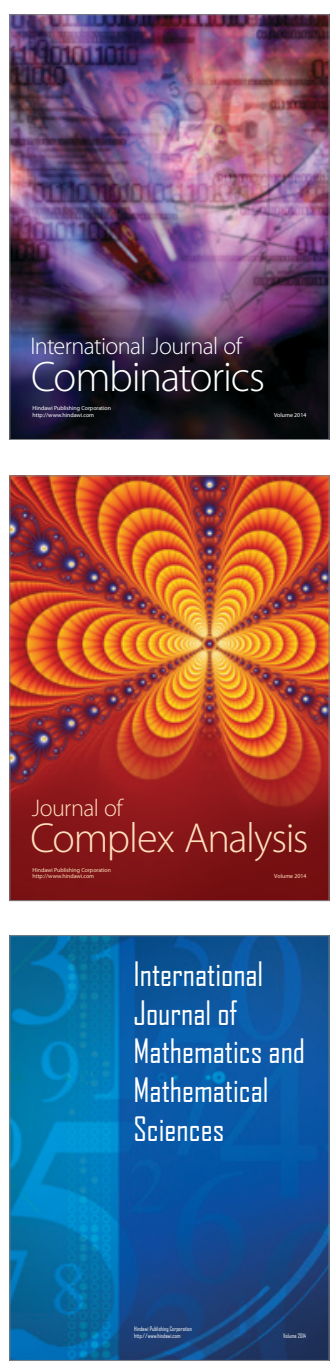
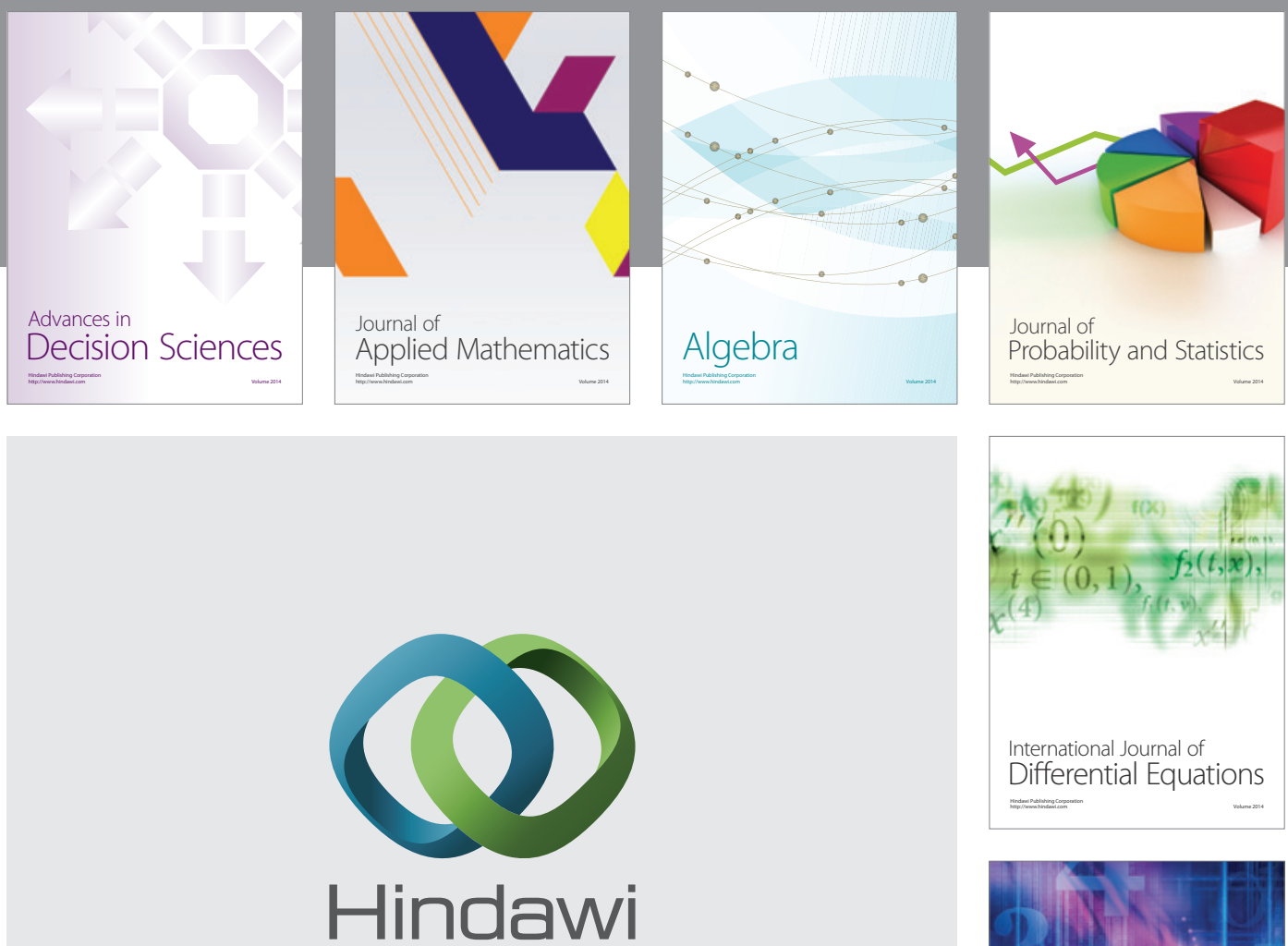

Submit your manuscripts at http://www.hindawi.com
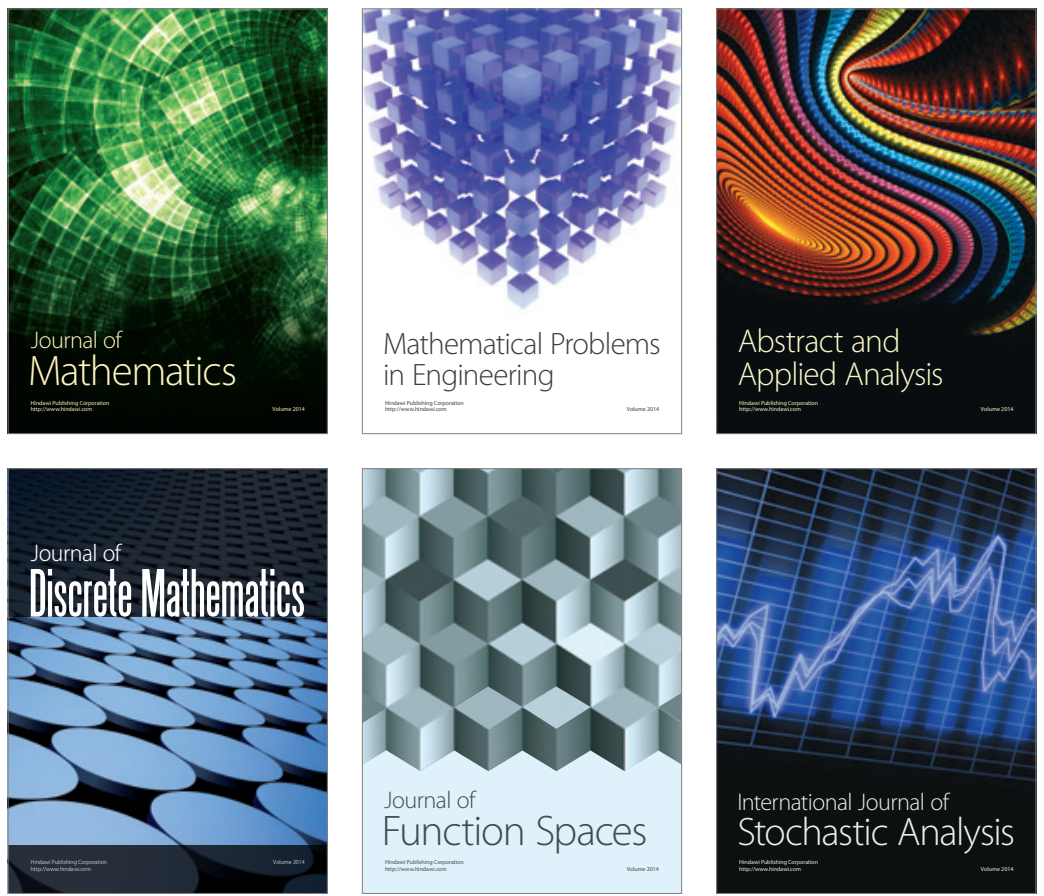

Journal of

Function Spaces

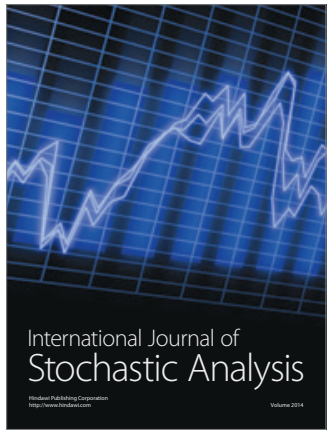

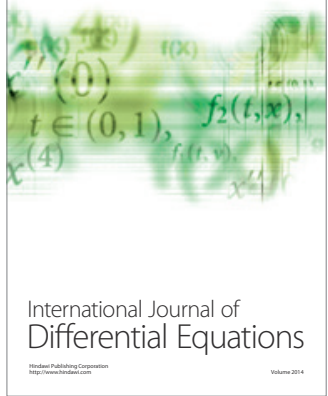
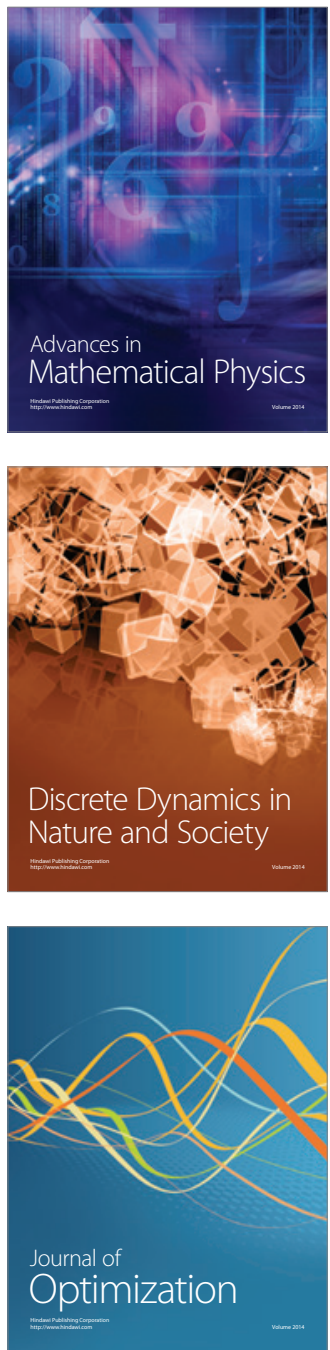